\title{
The effects of accumulated brisk walking on arterial stiffness in overweight adults and the level of support required to sustain adherence
}

\author{
T. M. Kearney ${ }^{1}$, A. M. Gallagher ${ }^{1}$, M. H. Murphy ${ }^{2}$, G. W. Davison ${ }^{2}$ and M. J. O'Kane ${ }^{3}$ \\ ${ }^{1}$ Northern Ireland Centre for Food and Health, University of Ulster, Coleraine BT52 1SA, UK, ${ }^{2}$ Sport and Exercise \\ Research Institute, University of Ulster, Newtownabbey BT37 OQB, UK and ${ }^{3}$ Department of Clinical Chemistry, \\ Altnagelvin Area Hospital, Londonderry BT47 6SB, UK
}

In recent years, arterial stiffness has been shown to be an independent marker for CVD and studies with adolescents indicate that individuals with an active lifestyle have lower arterial stiffness compared to sedentary individuals ${ }^{(1,2)}$. A few studies have been carried out investigating the effects of moderate exercise on arterial stiffness and data demonstrate that there is a negative correlation between physical activity and arterial stiffness. The aim of this study is to investigate the effects of accumulated brisk walking over a 6-month period on arterial stiffness measured by pulse wave velocity (PWV) in overweight adults. The effect of weekly contact $v$. monthly contact on compliance was also considered; the support provided was based on subjects being at the contemplative step of Prochaska and DiClementes transtheoretical model ${ }^{(3)}$. Individuals were contacted 4 months post intervention to enquire if they had continued to regularly walk.

Seventy seven overweight sedentary individuals (19 male, 58 female; age 45.6 years, SD 6.55; BMI 29.18, SD 4.27) participated in a randomised control trial and were allocated to one of the three groups: $n 25$ control group, $n 25$ walking with monthly contact group, $n 27$ walking with weekly contact group. The control group were given light non-cardiovascular exercises and were contacted monthly to control for any attention effects. The walking groups were asked to do three $10 \mathrm{~min}$ walks, on $5 \mathrm{~d}$ per week. Percentage body fat (Tanita scales), blood pressure, BMI $\left(\mathrm{kg} / \mathrm{m}^{2}\right)$, and PWV were measured at baseline and repeated after 6 months with follow-up measurements taken 4 months later. PWV was measured using a sensor-based device as described by McLaughlin et al. ${ }^{(4)}$ and a sub-maximal treadmill test was used to predict $\mathrm{VO}_{2}$ max at each of these three time-points.

Two-way ANOVA indicated no significant changes to BMI and percentage body fat. Blood pressure demonstrated a significant change $(P<0.05)$ with regard to trend, however, post-hoc tests showed no significant changes $(P>0.05)$. No significant changes were observed for predicted $\mathrm{VO}_{2} \max (P>0.05)$. PWV decreased significantly in both walking groups over the 6-month intervention period as compared to control and these beneficial effects were sustained beyond the end of the intervention period (i.e. 4 months follow-up) (see Figure).

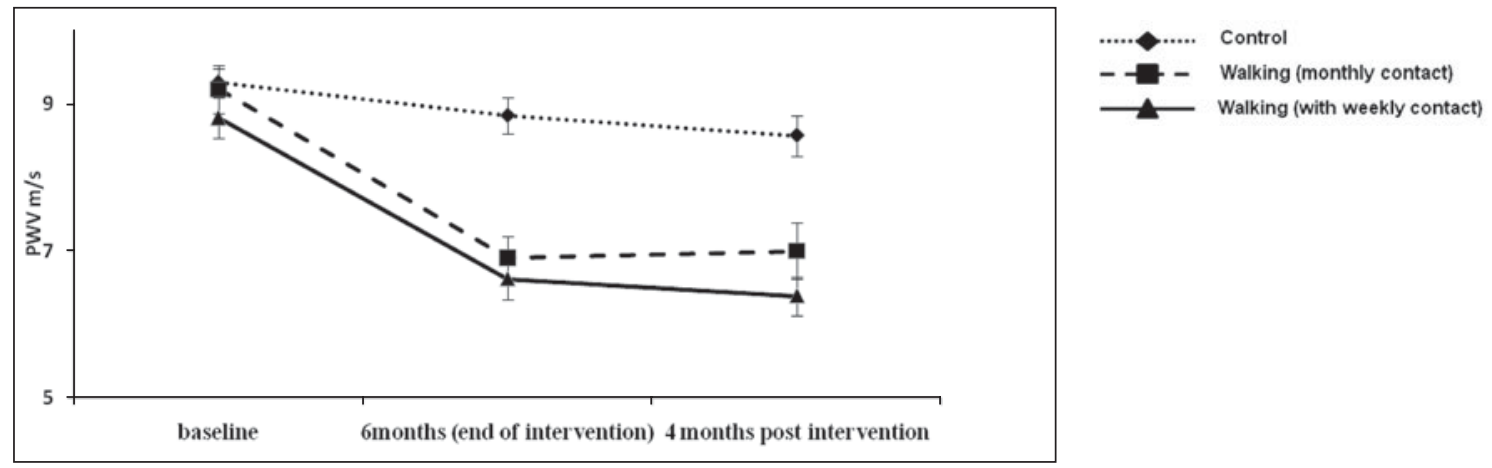

Values are means (SEM). At the baseline there was no significant differences between the three groups $(P>0.05)$, and over the duration of the study, the control did not change significantly $(P>0.05)$. However, at the end of the intervention and also at the follow-up stage, both the walking groups were significantly different from the control $(P<0.05)$, but were not significantly different from each other $(P>0.05)$. The results of this study confirm that accumulated walking on $5 \mathrm{~d}$ per week is sufficient to reduce PWV in overweight adults. Encouragement on a monthly basis was as successful at achieving these effects as on a weekly basis.

1. Boreham CA et al (2004) Hypertension 44, 721-726.

2. Ferreira I et al (2002) Eur J Clin Invest 32, 723-773.

3. Prochaska J \& DiClemente C. (1983) J Consulting Clin Psychol 51, 390-395.

4. McLaughlin et al (2003) Physiol Meas 24, 693-702. 